\title{
Efeitos da solução de continuidade do arco zigomático no crescimento facial em ratos jovens
}

\section{Effect of the discontinuity of the zygomatic arch on facial growth in young rats}

\author{
Luciane Ito de Queiroz* \\ Luciana Corrêa** \\ João Gualberto de Cerqueira Luz***
}

\begin{abstract}
RESUMO: Os resultados de estudos experimentais referentes ao arco zigomático na fase de crescimento, por meio de ressecções ou fraturas, são controversos. Assim, neste estudo foram avaliados experimentalmente os efeitos da solução de continuidade do arco zigomático no crescimento facial, portanto sem perdas teciduais ou desvios. Ratos com um mês de idade foram submetidos a um procedimento cirúrgico, para obtenção de uma solução de continuidade de um milímetro de espessura no arco zigomático do lado direito, tendo o lado esquerdo servido como controle. Os mesmos foram sacrificados com três meses de idade, sendo o crânio e as hemimandíbulas dissecados e radiografados. Foram feitas mensurações cefalométricas de dimensões da fossa infratemporal, da maxila e da mandíbula por meio de um sistema de computador. Não houve diferença significante entre os lados experimental e controle para as diferentes mensurações na maxila e na mandíbula. Foi verificada diferença significante para o comprimento ântero-posterior da fossa infra-temporal. Foi concluído que as conseqüências da solução de continuidade do arco zigomático no crescimento facial são localizadas apenas na própria estrutura.
\end{abstract}

DESCRITORES: Zigoma; Ossos faciais, crescimento e desenvolvimento; Ratos.

\begin{abstract}
The results of experimental studies on the zygomatic arch during the growth period, through excisions or fractures, are controversial. In this study, the effect of a discontinuity of the zygomatic arch on facial growth was experimentally evaluated, without tissual damage or deviations. One-month-old rats were submitted to a surgical procedure in order to obtain a one-milimeter-wide discontinuity of the right zygomatic arch. The left side served as a control. After three months, the rats were sacrificed, their skull and hemimandibles were dissected, and radiographs were obtained. Cephalometric measurements were carried out in order to assess the dimensions of the infratemporal fossa, maxilla and mandible, by means of a computer system. There was no significant difference between the experimental and control sides as to the measurements pertaining to the maxilla and mandible. Significant difference was observed in the anteroposterior length of the infratemporal fossa. It was concluded that the consequences of a discontinuity of the zygomatic arch on facial growth are restricted to the structure itself.
\end{abstract}

DESCRIPTORS: Zygoma; Facial bones, growth and development; Rats.

\section{INTRODUÇÃO}

O arco zigomático é considerado moderador do crescimento facial (Enlow ${ }^{3}$, 1990). Entretanto estudos experimentais que buscam determinar o papel desta estrutura no crescimento facial são poucos. Os resultados destes estudos, por meio de ressecções ou fraturas, são controversos. As ressecções parciais experimentais do arco zigomático não demonstram a ocorrência de alterações significantes no crescimento facial ${ }^{5,9,11}$. Entretanto, ocorrem alterações na maxila - maior no sentido anterior e menor no sentido transverso - quando é realizada a ressecção completa experimental do arco zigomático, havendo também assimetria quando unilateral ${ }^{2}$.

Na fratura experimental do arco zigomático em período de crescimento, com deslocamento medial do mesmo, a tendência é o retorno à posição original desta estrutura, havendo duas alterações significantes: menor profundidade da fossa infratemporal e desvio da mandíbula para o lado da fratura $^{4,8}$. Também, nesse mesmo modelo, diminuições significantes a menor são verificadas para a altura do corpo e do ramo da mandíbula ${ }^{10}$. Quando realizada de modo bilateral, além destes acha-

\footnotetext{
*Aluna de Iniciação Científica (FUNDECTO) - Disciplina de Traumatologia Maxilo-Facial do Departamento de Cirurgia, Prótese e Traumatologia Maxilo-Faciais; **Mestre e Doutora em Patologia Bucal; ***Professor Associado - Disciplina de Traumatologia Maxilo-Facial do Departamento de Cirurgia, Prótese e Traumatologia Maxilo-Faciais - Faculdade de Odontologia da Universidade de São Paulo.
} 
Queiroz LI de, Corrêa L, Luz JG de C. Efeitos da solução de continuidade do arco zigomático no crescimento facial em ratos jovens. Pesqui Odontol Bras 2002;16(4):374-378.

dos, ocorre encurtamento no sentido ântero-posterior do terço médio da face ${ }^{1}$. A tendência de retorno do arco zigomático à sua posição original também foi observada diante de procedimento experimental que promoveu deslocamento lateral do mesmo ${ }^{12}$.

A proposta deste trabalho foi avaliar os efeitos da solução de continuidade do arco zigomático no crescimento facial, portanto sem perdas teciduais ósseas ou desvios.

\section{MATERIAL E MÉTODOS}

Para este estudo foram utilizados 25 ratos Wistar com um mês de idade, com peso inferior a 100 gramas. O manuseio dos animais foi de acordo com as normas propostas pela Sociedade Brasileira de Experimentação Animal, tendo o trabalho sido aprovado pelo Comitê de Ética da instituição.

Todos os espécimes foram submetidos à mesma intervenção cirúrgica. Os animais foram submetidos a anestesia geral com o emprego de cloridrato de xilidino di-hidrotiazina (Rompum, Bayer) na dose de $0,8 \mathrm{mg} / \mathrm{kg}$ de peso e de cloridrato de cetamina (Francotar, Francodex) na dose de $10 \mathrm{mg} / 100 \mathrm{~g}$ de peso corporal, ambos medicamentos aplicados por via intraperitonial.

Em seguida foi realizada tricotomia na região pré-auricular do lado direito e anti-sepsia com solução de polivinilpirrolidona-iodo. O acesso à estrutura foi obtido por meio de uma incisão pré-auricular, paralelamente ao arco zigomático, seguida de divulsão romba e sua identificação. A solução de continuidade, realizada na parte média do arco zigomático direito, foi obtida com emprego de broca número 702 de "carbide" em motor de baixa rotação, sob irrigação com soro fisiológico. O ato cirúrgico foi concluído com sutura por planos com fio de "nylon" monofilamentar 4.0. O lado esquerdo serviu como controle.

A seguir, os animais foram alojados em gaiolas adequadas, recebendo ração granulada para roedores, fragmentada nos primeiros 15 dias e água ad libitum. Os animais foram sacrificados após um período de 60 dias por dose letal de anestésico geral. Após o sacrifício, suas cabeças foram removidas, sua pele e demais tecidos moles foram retirados, a mandíbula foi desarticulada e as hemimandíbulas separadas pela sínfise fibrosa. Ambos, hemimandíbulas e crânio foram fixados em formol tamponado a $10 \%$.

Foram feitas radiografias do crânio em tomada axial e das hemimandíbulas em tomada lateral. Foi utilizado filme radiográfico do tipo periapical (Ektaspeed, Kodak) e aparelho de raios X odonto- lógico (Spectro II, Dabi Atlante) no regime de $56 \mathrm{kVp}, 10 \mathrm{~mA}$ e $0,4 \mathrm{~s}$ para o crânio e $0,3 \mathrm{~s}$ para as hemimandíbulas. Essas imagens radiográficas foram digitalizadas no Laboratório de Informática Dedicado à Odontologia (LIDO), por meio do scanner HP Desk Scan com tampa para slides Scan Jet 4c/T, acoplado a um microcomputador Pentium 166 e monitor MultiSync M700. As imagens digitalizadas foram processadas no software Adobe Photoshop ${ }^{\circledast}$ para padronização do brilho e do constraste. A mensuração e quantificação de dimensões da fossa infratemporal, da maxila e da mandíbula, descritas a seguir, foram feitas por intermédio do software Imagelab ${ }^{\circledR}$.

Para a tomada radiográfica axial do crânio, tanto do lado direito como do esquerdo, foram feitas cinco mensurações: comprimento ântero-posterior da fossa infratemporal (AF-PF); distância entre a bula timpânica e a raiz mesial do primeiro molar superior (BT-RM); distância entre a bula timpânica e o forame infraorbitário (BT-FI); distância entre o forame infraorbitário e o ponto incisal (FI-PI); distância entre o ponto incisal e a raiz mesial do primeiro molar superior (PI-RM) (Figura 1).

As mensurações que se referem à altura das hemimandíbulas radiografadas na norma lateral, tanto do lado direito como do esquerdo, foram as seguintes: distância entre o processo condilar e o processo angular da mandíbula (PC-PA) e distância da intersecção da face distal do terceiro molar com o ramo da mandíbula e a incisura antegônica (TM-IA). Considerou-se como incisura antegônica

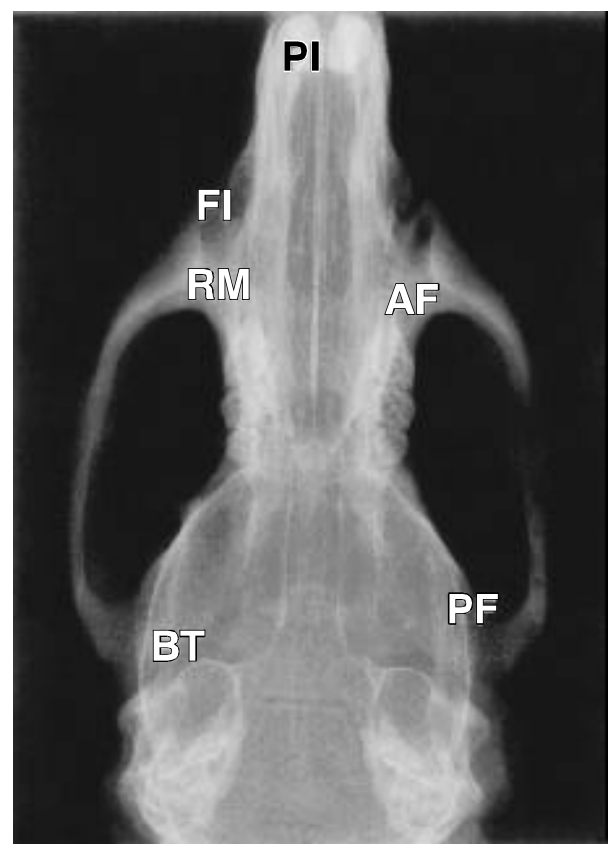

FIGURA 1 -

Radiografia axial do crânio. $\mathrm{AF}=$ ponto anterior da fossa infratemporal; $\mathrm{PF}=$ ponto posterior da fossa infratemporal; $\mathrm{BT}=$ bula timpânica; $\mathrm{RM}=$ raiz mesial do primeiro molar; $\mathrm{FI}=$ forame infraorbitário; $\mathrm{PI}=$ ponto incisal. 
Queiroz LI de, Corrêa L, Luz JG de C. Efeitos da solução de continuidade do arco zigomático no crescimento facial em ratos jovens. Pesqui Odontol Bras 2002;16(4):374-378.

ou incisura pré-angular o entalhe presente na base da mandíbula, de localização anterior ao ângulo da mandíbula. As mensurações que se referem ao comprimento das hemimandíbulas foram: distância entre a inserção ou articulação do incisivo no osso e o processo angular (II-PA) e distância entre a inserção ou articulação do incisivo no osso e o processo condilar (II-PC) (Figura 2).

Foi utilizado o teste $t$ de Student para a verificação da significância da diferença entre os valores médios dos lados direito (experimental) e esquerdo (controle). Foi fixado o nível de significância de 5\% para todas as análises estatísticas.

\section{RESULTADOS}

Não foram observadas alterações macroscópicas na face dos animais, sendo a seguir, descritas as mensurações obtidas.

\section{Comprimento ântero-posterior da fossa infratemporal}

Os valores médios do comprimento ântero-posterior da fossa infra-temporal, para os lados direito e esquerdo, são observados na Tabela 1. Com a aplicação do teste $t$ de Student foi constatada diferença significante a maior ao nível de $1 \%$.

\section{Distância entre a bula timpânica e a raiz mesial do primeiro molar superior}

Os valores médios da distância entre a bula timpânica e a raiz mesial do primeiro molar superior, para os lados direito e esquerdo, são observados na Tabela 1. Com a aplicação do teste $t$ de Student foi constatada diferença não significante.

\section{Distância entre a bula timpânica e o forame infra-orbitário}

Os valores médios da distância entre a bula timpânica e o forame infra-orbitário, para os lados direito e esquerdo, são observados na Tabela 1 . Com a aplicação do teste $t$ de Student foi constatada diferença não significante.

TABELA 1 - Mensurações obtidas por meio da radiografia axial. Valores em milímetros.

\begin{tabular}{l|c|c|c|c}
\hline \multirow{2}{*}{$\begin{array}{l}\text { Lado } \\
\text { Mensuração }\end{array}$} & \multicolumn{2}{|c|}{$\begin{array}{c}\text { Direito } \\
\text { (experimental) }\end{array}$} & \multicolumn{2}{c}{$\begin{array}{c}\text { Esquerdo } \\
\text { (controle) }\end{array}$} \\
\cline { 2 - 5 } AF-PF & Média & $\begin{array}{c}\text { Desvio } \\
\text { padrão }\end{array}$ & Média & $\begin{array}{c}\text { Desvio } \\
\text { padrão }\end{array}$ \\
\hline BT-RM & 14,78 & 0,45 & 14,43 & 0,47 \\
\hline BT-FI & 17,04 & 0,58 & 16,88 & 0,63 \\
\hline FI-PI & 20,95 & 0,58 & 20,81 & 0,61 \\
\hline PI-RM & 7,89 & 0,30 & 7,88 & 0,37 \\
\hline \hline
\end{tabular}

$\mathrm{AF}=$ ponto anterior da fossa infratemporal; $\mathrm{PF}=$ ponto posterior da fossa infratemporal; $\mathrm{BT}=$ bula timpânica; $\mathrm{RM}=$ raiz mesial do primeiro molar; $\mathrm{FI}=$ forame infra-orbitário; PI = ponto incisal.
FIGURA 2 - Radiografia lateral das hemimandíbulas. $\mathrm{PC}=$ processo condilar; $\mathrm{PA}=$ processo angular; $\mathrm{TM}=$ face distal do terceiro molar; IA = incisura antegônica; II = inserção do incisivo.

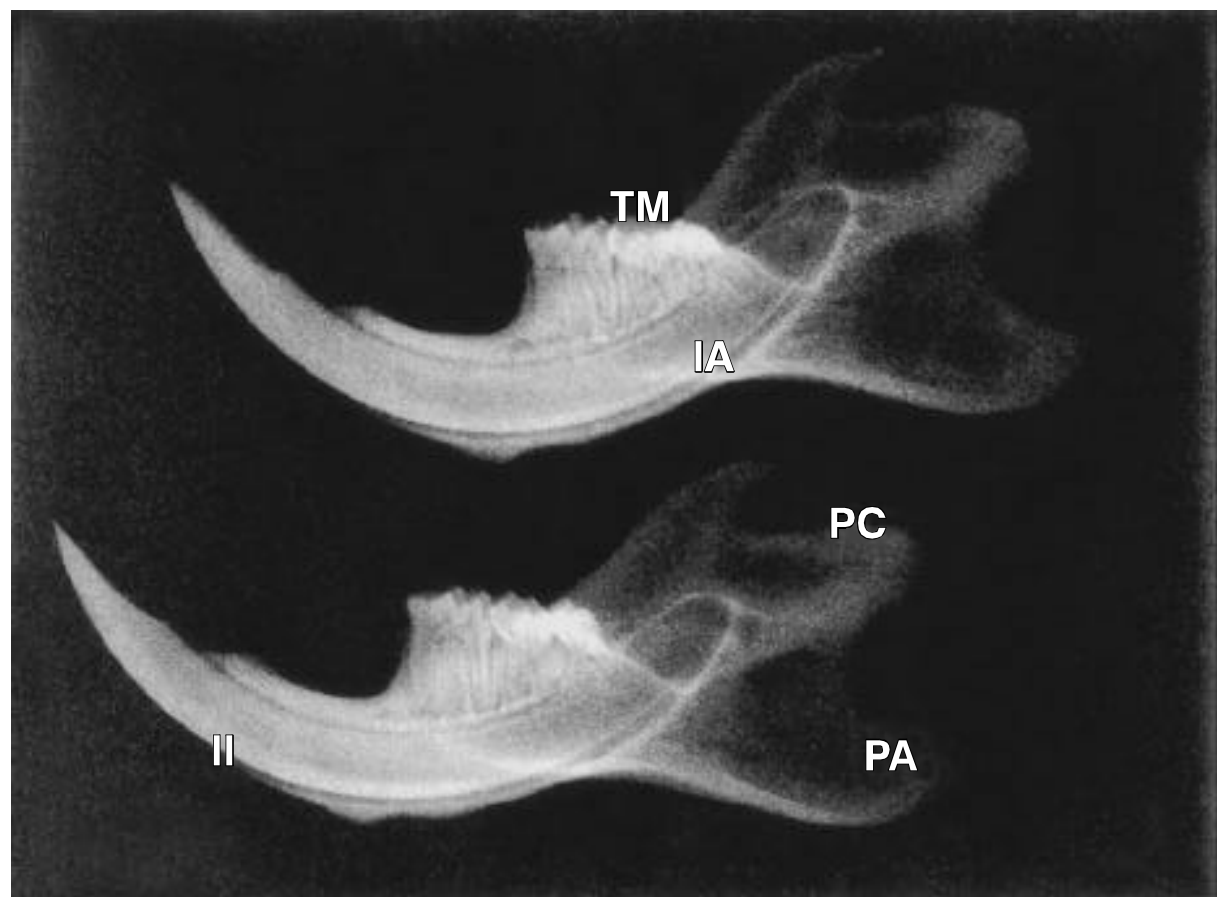


Queiroz LI de, Corrêa L, Luz JG de C. Efeitos da solução de continuidade do arco zigomático no crescimento facial em ratos jovens. Pesqui Odontol Bras 2002;16(4):374-378.

\section{Distância entre o forame infra-orbitário e o ponto incisal}

Os valores médios da distância entre o forame infra-orbitário e o ponto incisal, para os lados direito e esquerdo, são observados na Tabela 1 . Com a aplicação do teste $t$ de Student foi constatada diferença não significante.

\section{Distância entre o ponto incisal e a raiz mesial do primeiro molar superior}

Os valores médios da distância entre o ponto incisal e a raiz mesial do primeiro molar superior, para os lados direito e esquerdo, são observados na Tabela 1. Com a aplicação do teste $t$ de Student foi constatada diferença não significante.

\section{Distância entre o processo condilar e o processo angular da mandíbula}

Os valores médios da distância entre o processo condilar e o processo angular da mandíbula, para os lados direito e esquerdo, são observados na Tabela 2. Com a aplicação do teste $t$ de Student foi constatada diferença não significante.

\section{Distância da intersecção da face distal do terceiro molar com o ramo da mandíbula e a incisura antegônica}

Os valores médios da distância da intersecção da face distal do terceiro molar com o ramo da mandíbula e a incisura antegônica, para os lados direito e esquerdo, são observados na Tabela 2 . Com a aplicação do teste $t$ de Student foi constatada diferença não significante.

\section{Distância entre a inserção do incisivo e o processo angular}

Os valores médios da distância entre a inserção do incisivo no osso e o processo angular, para os lados direito e esquerdo, são observados na Tabela 2. Com a aplicação do teste $t$ de Student foi constatada diferença não significante.

\section{Distância entre a inserção do incisivo e o processo condilar}

Os valores médios da distância entre a inserção do incisivo no osso e o processo condilar, para os lados direito e esquerdo, são observados na Tabela 2. Com a aplicação do teste $t$ de Student foi constatada diferença não significante.
TABELA 2 - Mensurações obtidas por meio da radiografia das hemimandíbulas. Valores em milímetros.

\begin{tabular}{l|c|c|c|c}
\hline \multirow{2}{*}{ Lado } & \multicolumn{2}{|c|}{$\begin{array}{c}\text { Direito } \\
\text { (experimental) }\end{array}$} & \multicolumn{2}{c}{$\begin{array}{c}\text { Esquerdo } \\
\text { (controle) }\end{array}$} \\
\cline { 2 - 5 } Mensuração & Média & $\begin{array}{c}\text { Desvio } \\
\text { padrão }\end{array}$ & Média & $\begin{array}{l}\text { Desvio } \\
\text { padrão }\end{array}$ \\
\hline PC-PA & 7,07 & 0,40 & 7,26 & 0,32 \\
\hline TM-IA & 6,22 & 0,25 & 6,16 & 0,25 \\
\hline II-PA & 23,66 & 0,79 & 23,34 & 0,70 \\
\hline II-PC & 23,96 & 0,67 & 23,69 & 0,63 \\
\hline \hline
\end{tabular}

$\mathrm{PC}=$ processo condilar; $\mathrm{PA}=$ processo angular; $\mathrm{TM}=$ face distal do terceiro molar; IA = incisura antegônica; II = inserção do incisivo.

\section{DISCUSSÃO}

Neste estudo analisaram-se os efeitos da solução de continuidade do arco zigomático no crescimento facial em ratos jovens. Os animais mantiveram atividade mastigatória e apresentaram ganho de peso após o procedimento cirúrgico. Embora não tenha sido utilizado grupo controle, este achado mostra que os animais suportaram bem o procedimento realizado. Foram utilizados ratos com um mês de idade, faixa etária classificada como infantil. Assim, foi possivel acompanhar o crescimento até a fase adulta aos três meses de idade, assim como encontrado nos trabalhos de Goulart et $a .^{4}{ }^{4}$ (1998) e Rocha et al. ${ }^{10}$ (1999).

Alterações significantes, representadas pelo maior comprimento ântero-posterior da fossa infratemporal, foram observadas. Esse dado é semelhante ao observado em experimento promovendo a ressecção completa do arco zigomático, embora com alterações bem mais discretas ${ }^{2}$. Sua ressecção provoca um aumento da projeção facial e diminuição da largura transversa da face no lado comprometido ${ }^{2}$.

Não foram encontradas alterações significantes nas demais mensurações faciais, nem assimetria facial foi observada macroscopicamente no presente estudo. Esse dado está de acordo com alguns trabalhos prévios, discutidos a seguir. Ressecções parciais experimentais do arco zigomático não têm demonstrado alterações significantes no crescimento facial ${ }^{5,9,11}$. Outro estudo em que foi feita a ressecção parcial do arco zigomático, junto à sua articulação no osso temporal, evidenciou alterações funcionais articulares seguidas de remodelamento das superfícies lateral e anterior do côndilo mas não houve referência ao crescimento facial ${ }^{6}$. 
Queiroz LI de, Corrêa L, Luz JG de C. Efeitos da solução de continuidade do arco zigomático no crescimento facial em ratos jovens. Pesqui Odontol Bras 2002;16(4):374-378.

Tendo em vista os resultados deste trabalho verificou-se que as conseqüências da solução de continuidade do arco zigomático no crescimento facial são localizadas apenas na própria estrutura. Isso difere do observado diante da fratura experimental do arco zigomático em modelo utilizando ratos e o mesmo período de observação, quando ocorreram alterações a menor na fossa infratemporal, na altura da mandíbula e no comprimento da ma $x^{x i l a}{ }^{1,4,8,10}$. Por outro lado, tais alterações mais intensas podem estar associadas ao comprometimento funcional, não intencional, dos músculos associados, em especial do temporal ${ }^{7,10}$.

\section{CONCLUSÕES}

Nas condições desta pesquisa, podemos concluir que:

\section{REFERÊNCIAS}

1. Bittar AA, Rabeque FC, Novelli MD, Luz JGC. Efeitos da fratura bilateral do arco zigomático no crescimento facial em ratos jovens. RPG Rev Pós Grad 1999;6:147-52.

2. Del Campo AF, Eliozondo MM, Magnelli LM, Valdadez AS, Ontiveros DS. Craniofacial development in rats with early resection of the zygomatic arch. Plast Reconstr Surg 1995;95:486-95.

3. Enlow DH. Facial growth. $3^{\text {rd }}$ ed. Philadelphia: Saunders; 1990.

4. Goulart AC, Fernandes EMV, Cavalcanti FC, Novelli MD, Luz JGC. Fratura do arco zigomático no período de crescimento: estudo experimental em ratos. Rev Odontol Univ São Paulo 1998;12:75-80.

5. Jacobs B, Kronman JH. The zygomatic arch and its possible influence on craniofacial growth and development. Angle Orthodont 1977;47:136-40.

6. Kotyla JR. An exploratory study of the mandibular condyle in the rat following changes in articular function. Eur $J$ Orthod 1986;8:265-70.
- houve diferença significante, com maiores valores, para o comprimento ântero-posterior da fossa infratemporal;

- não houve diferença significante para as diversas mensurações realizadas na maxila;

- não houve diferença significante para as diversas mensurações realizadas na mandíbula;

- as conseqüências da solução de continuidade do arco zigomático no crescimento facial foram localizadas apenas na própria estrutura.

\section{AGRADECIMENTOS}

À Fundação para o Desenvolvimento Científico e Tecnológico da Odontologia (FUNDECTO) pela concessão de Bolsa de Iniciação Científica. Ao Prof. Dr. Moacyr Domingos Novelli, pelo auxílio nas mensurações cefalométricas por sistema de computador.

7. Mierzwa J, Schumacher GH, Fanghanel J, Koster D. Craniofacial growth under the influence of the blood supply. 6 - metric studies of the skull. Anat Anz 1997; 163:389-99.

8. Pastore GP, Andrade JOC, Luz JGC. Fratura unilateral do arco zigomático em ratos jovens após diferentes períodos. RPG Rev Pós Grad 2000;7:108-13.

9. Ritsilã MD, Alhopuro S, Ranta R. The role of the zygomatic arch in the growth of the skull in rabbits. Proc Finn Dent Soc 1973;69:164-7.

10. Rocha EMVF, Goulart AC, Novelli MD, Luz JGC. Efeitos da fratura do arco zigomático no crescimento facial em ratos jovens. Rev Odontol Univ São Paulo 1999;13:37-41.

11. Umemura S. Experimental study of the influence of removal of the zygomatic arch on craniofacial growth and development. Shikwa Gakuho 1989;89:981-1019.

12. Vargervik K, Farias M, Ousterhout D. Changes on zygomatic arch position following experimental lateral displacement. J Cranio-Max-Fac Surg 1987;15:208-12. 